\title{
Avaliação da Aprendizagem em Ambientes Educacionais: Uma Revisão Sistemática
}

\author{
Alane de Almeida Ferreira, Vera Maria Benjamin Werneck, Neide dos Santos \\ Programa de Pós-Graduação em Ciências Computacionais \\ Instituto de Matemática e Estatística - UERJ - Rio de Janeiro - RJ - Brazil \\ alane \{vera, neide\}@ime.uerj.br
}

\begin{abstract}
The paper presents a systematic review on evaluation of Virtual Learning Environments in order to identify methods and techniques used in VLE uses' evaluation. The work describes the review steps and presents the results. From the final selection of 46 articles found in literature sources, widely consulted by computers in education community, it appears that there is a tendency to build evaluation models based on questionnaires and the use of pre and post-tests. Most studies show a positive impact on VLE use. Some of them present variables that can hinder the results. These variables impact in AVA-based learning process needs to be better investigated.
\end{abstract}

Resumo. Este artigo descreve uma revisão sistemática sobre a avaliação de Ambientes Virtuais de Aprendizagem para identificar métodos e técnicas utilizadas na avaliação do uso desses ambientes. A partir da seleção final de 46 artigos, encontrados em fontes de referência amplamente consultadas pela comunidade de informática na educação, verifica-se tendência na construção de modelos de avaliação, baseados em questionários e no uso de pré e pós testes. Muitos trabalhos apontam impacto positivo no uso dos AVAs e alguns apresentam variáveis que podem comprometer os resultados. $O$ impacto dessas variáveis no processo de aprendizagem mediado por AVAs precisa ser melhor investigado.

\section{Introdução}

Os ambientes virtuais de aprendizagem (AVA) vêm sendo usados há mais de uma década com o propósito específico de ensinar tópicos curriculares e/ou desenvolver determinadas competências, complementando o processo de ensino uma vez que é possível encontrar uma diversidade destes. No entanto, a avaliação de sua efetividade como instrumentos de apoio a ganhos de aprendizagem não tem sido objeto de muitos estudos. Segundo Galvão et al. (2013), uma primeira exigência para se realizar um processo de avaliação é que o ambiente possibilite interação entre o usuário e o objeto de estudo. Portanto, para desenvolvimento do usuário, a interação deve ir além de uma possível escolha dentre opções disponíveis.

$\mathrm{Na}$ busca de tornar o processo de ensino e aprendizagem mais atrativo e eficaz para os alunos, diversas formas de ensino têm sido exploradas. Cada vez mais jogos educacionais têm sido elaborados e se mostrado uma técnica potencialmente eficaz de aprendizado [Shukor et al, 2015]. Os alunos apresentam melhor absorção do conteúdo 
V Congresso Brasileiro de Informática na Educação (CBIE 2016)

Anais do XXVII Simpósio Brasileiro de Informática na Educação (SBIE 2016)

quando este é vinculado a aulas mais dinâmicas, promovendo resultado e diversão [Mattos e Costa, 2013].

Apesar do potencial atrelado ao uso desses ambientes virtuais educacionais, pouco se tem feito para avaliar sua real efetividade. Ainda não se conhece o quanto os jogos contribuem na educação [Savi et al, 2011]. A maioria dos trabalhos voltados para avaliação destacam ou o processo de desenvolvimento do ambiente virtual ou a avaliação do mesmo em relação a aspectos como interface, qualidade, clareza abstendose de tratar sobre o real ganho no ensino.

Neste contexto, realizou-se uma revisão sistemática da literatura para identificar os métodos e técnicas utilizadas na avaliação do uso de ambientes virtuais de aprendizagem. Pretendeu-se buscar resultados de avaliações já implementadas com o intuito de que esta revisão sistemática subsidie o modelo a ser proposto em trabalho futuro. Analisou-se também, a experiência do usuário visando ampliar o campo de estudo sobre este tema.

Este artigo tem por objetivo apresentar uma revisão sistemática da literatura no domínio da avaliação dos ambientes virtuais de aprendizagem, buscando identificar um estado da arte, capaz de fornecer subsídios a pesquisadores/desenvolvedores envolvidos na área de avaliação de AVA, bem como oferecer elementos para a construção de novos modelos de avaliação. Com este propósito, o artigo é composto de 4 seções, além desta introdução. A seção 2 brevemente descreve os trabalhos correlatos; a seção 3 apresenta a metodologia adotada na revisão sistemática da literatura; a seção 4 discute os resultados obtidos; e por fim, a seção 5 oferece as conclusões do trabalho.

\section{Trabalhos Correlatos}

Na busca da qualidade no processo de ensino e aprendizagem, métodos avaliativos têm sido cada vez mais aperfeiçoados. Venancio \& Lopes (2013) faz uma revisão de artigos publicados no SBIE, WIE e WAvalia. Seu principal foco é nos artigos que utilizam a avaliação formativa tanto em ambientes virtuais quanto de forma presencial.

Souza et al (2013) propõe a aplicação de uma técnica da estatística multivariada em dados educacionais inerentes ao processo de avaliação de aprendizagem de um ambiente virtual. A proposta dos autores é avaliar as possíveis relações existentes entre as disciplinas e mostrar o quanto o currículo do curso pode influenciar nos parâmetros da avaliação.

No trabalho de Galdino et al (2014) apresenta-se uma avaliação da aprendizagem do ponto de vista dos materiais disponíveis no ambiente virtual de aprendizagem. Concluiu-se que a maneira como o material didático é disponibilizado influencia diretamente no processo de ensino e aprendizagem.

Em Gomes et al (2015) é mostrada uma avaliação de um jogo educativo para o ensino do pensamento computacional sob dois vieses, o da avaliação formativa e o da avaliação objetiva. A avaliação objetiva preocupou-se com vários aspectos, como por exemplo, facilidade de uso, design de tela, afetividade, entre outros. A avaliação formativa observou aspectos como motivação e compreensão de alguns elementos e conceitos do jogo. 
V Congresso Brasileiro de Informática na Educação (CBIE 2016)

Anais do XXVII Simpósio Brasileiro de Informática na Educação (SBIE 2016)

Keller (2010) concebeu o modelo ARCS (Atenção, Relevância, Confidência, Satisfação) que tem como objetivo empregar estratégias motivacionais no projeto de materiais educacionais. Este modelo pode ser utilizado para avaliar a motivação de estudantes quando utilizarem materiais educacionais.

Kirkpatrick (1996) desenvolveu um modelo de treinamento composto por quatro níveis. Estes avaliam a reação, aprendizagem, comportamento e resultados. Este modelo também pode ser usado para avaliar materiais instrucionais por meio dos níveis propostos.

Savi (2011a) propõe um modelo para avaliação de jogos educacionais baseado no modelo de avaliação de treinamentos de Kirkpatrick, nas estratégias motivacionais do modelo Arcs de Keller, na experiência do usuário e na taxonomia de Bloom.

\section{Revisão Sistemática da Literatura}

A revisão sistemática da literatura (SLR) é utilizada para identificar, analisar e interpretar todos as evidencias disponíveis relacionadas a uma pesquisa específica [Wholin et al, 2012].

As principais fases para realização de uma SVR são o planejamento da revisão, condução da revisão e apresentação dos resultados [Kitchenham, 2007]. Um protocolo de pesquisa foi produzido durante a fase de planejamento para realização dessa revisão sistemática. Nesta seção, apresenta-se esse protocolo elaborado a partir das atividades descritas.

\subsection{Questão de pesquisa}

A questão de pesquisa é decisiva e orienta todo o processo de revisão pois é a partir dela que serão definidas as strings, os dados que serão coletados e a análise dos mesmos de modo que a questão possa ser respondida.

Pensando no objetivo principal desta revisão sistemática que é identificar métodos de avaliação da aprendizagem em ambientes virtuais, definiu-se as seguintes questões de pesquisa apresentadas na Tabela 1.

Tabela 1. Questões da Pesquisa da Revisão de Avaliação da Aprendizagem em Ambientes Virtuais

\begin{tabular}{|l|l|}
\hline & \multicolumn{1}{|c|}{ Descrição das Questões } \\
\hline Q1 & Que tipos de avaliações estão sendo implementadas? \\
\hline Q2 & Em que ano foram desenvolvidas? \\
\hline Q3 & Em que área estas avaliações têm sido desenvolvidas? \\
\hline Q4 & Que tipos de ferramentas têm sido utilizados? \\
\hline Q5 & Em que autor a proposta de avaliação foi fundamentada? \\
\hline Q6 & Qual é o público destinado a utilizar estes ambientes virtuais? \\
\hline Q7 & Em quanto tempo essas avaliações foram desenvolvidas? \\
\hline Q8 & Quão satisfatórios são os resultados destas avaliações? \\
\hline
\end{tabular}

\subsection{Termos da busca}

Os termos de busca para realização da pesquisa incluem avaliação (evaluation) por se tratar de avaliação, aprendizagem, pois essa avaliação é focada na aprendizagem em ambientes virtuais (jogos, softwares educacionais, entre outros). A string de busca definida para a realização da pesquisa foi considerada tanto em português como inglês: 
V Congresso Brasileiro de Informática na Educação (CBIE 2016)

Anais do XXVII Simpósio Brasileiro de Informática na Educação (SBIE 2016)

("avaliação" OR "evaluation" OR “efetividade” OR "effectiveness" OR "análise" OR "analysis")

AND (aprendizagem OR learning OR conhecimento OR knowledge OR experiência OR experience)

AND (jogos educacionais OR educational games OR ambientes virtuais OR virtual environments OR educação OR education OR educação à distância OR distance learning OR software educacionais OR educational software).

\subsection{Fonte de Dados}

A busca dos artigos foi realizada nas bibliotecas relevantes da área de informática na educação. As seguintes fontes foram pesquisadas: Science Direct, Google Scholar, IEEExplore, ACM Digital library, Springerlink, Higher Education, Citeseerx, Scielo, Portal Capes, Congresso Brasileiro de Informática na Educação (CBIE), Simpósio Brasileiro de Informática na Educação (SBIE), Workshop de Informática na Escola (WIE), Revista Brasileira de Informática na Educação (RBIE), Workshop sobre Educação em Computação (WEI), Jornada de Atualização em Informática na Educação (JAIE), Workshop de Desafios da Computação Aplicada à Educação (DESAFIE), Fórum de Educação em Engenharia de Software (CBSOFT/FEES), Grupo de Informática Aplicada à Educação (GINAPE) do NCE/UFRJ, Laboratório de Tecnologias da Informação e Comunicação da Universidade Federal do Rio de Janeiro (LATEC UFRJ) e Programa de Pós-Graduação em Informática da Universidade Federal do Estado do Rio de Janeiro (PPGI/UNIRIO).

\subsection{Realização de Busca}

O processo de busca dos artigos considerou as fontes de dados citadas no período de março a abril de 2016, sendo revisados em maio de 2016. Os critérios de inclusão e exclusão estabelecidos foram baseados nas questões de pesquisa. Critérios de inclusão: (i) o artigo deve estar escrito em Português ou Inglês. (ii) artigos de 2010 até a presente data, e (iii) todos os artigos que desenvolvem, avaliam ou descrevem algum processo de avaliação de aprendizagem com ambientes virtuais. Critérios de Exclusão: (i) o artigo deve estar disponível para acesso livre em bibliotecas digitais. (ii) a existência de um artigo mais recente ou mais completo (tratando do mesmo trabalho) elimina o artigo anterior. (iii) artigos que descrevem avaliação de jogos sem foco na aprendizagem. (iv) artigos que avaliam aspectos que saem do escopo do ensino (afetividade, comportamento, ...). (v) artigos que não descrevem a avaliação da aprendizagem através de um jogo ou ambiente específico.

\subsection{Seleção dos Estudos e Extração dos Dados}

Realizou-se uma busca por trabalhos no período de 6 anos, 2010 a 2016. Para selecionar os trabalhos foram utilizados os critérios de inclusão e exclusão baseados nas questões de pesquisa. Tais critérios foram estabelecidos para permitir a seleção adequada dos artigos. Baseado em Peixoto (2015), foram executados os seguintes passos: (i) análise dos títulos, (ii) análise dos resumos, (iii) análise rápida do texto, (iv) leitura detalhada e (v) referência. A análise dos títulos tem como intuito eliminar trabalhos duplicados e irrelevantes, por isso apenas os títulos dos artigos encontrados são analisados. A seguir, é realizada a leitura dos resumos para restringir a lista e depois elaborada uma análise rápida dos artigos. 
V Congresso Brasileiro de Informática na Educação (CBIE 2016)

Anais do XXVII Simpósio Brasileiro de Informática na Educação (SBIE 2016)

Com uma amostra ainda menor são analisados os artigos através da completa leitura desses e após são verificadas as referências bibliográficas incorporando a lista artigos relevantes através da aplicação dos critérios de inclusão e exclusão estabelecidos.

\section{Resultados}

Ao se realizar a busca foram encontrados 302 artigos. Na etapa de análise dos títulos etapa foram eliminados 66 artigos, restando 237 artigos. Ao se analisar os resumos dos 237 artigos descartaram-se 126 artigos e restaram 111 artigos. Ao realizar uma leitura rápida dos artigos, eliminou-se 57 artigos que não atendiam aos critérios de inclusão e exclusão, restando 54 artigos. Esses artigos foram lidos na íntegra e depois disso excluiu-se mais 17 artigos considerando os critérios de exclusão, ficando com uma lista 37 artigos. Dos 37 artigos restantes, analisaram-se as referências bibliográficas e surgiram 24 novos artigos. Utilizando os critérios de inclusão e exclusão, excluiu-se mais 15 artigos, restando nove. No total foram 37 artigos somados aos nove, resultando em 46 artigos.

A revisão sistemática incluiu trabalhos desenvolvidos no período de 2010 até a presente data. Os artigos encontrados apresentam distribuição crescente e a partir de 2012, uniforme em relação ao ano de desenvolvimento, sendo que 2015 foi ano em que existiram mais trabalhos em avaliação de aprendizagem em AVA (Figura 1).

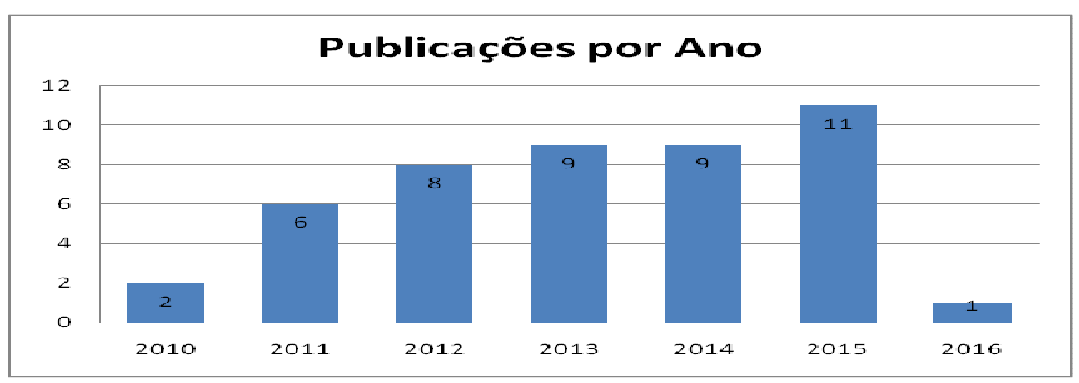

Figura 1. Distribuição das Publicações por Ano

Os 46 artigos descrevem a avaliação da aprendizagem em ambientes virtuais sendo muitos desenvolvidos por pesquisadores do Brasil, seguidos por Taiwan, EUA, Turquia, Malásia, Irã entre outros (Figura 2). Mais da metade (60\%) destes artigos foram encontrados nas fontes Elsevier Science Direct, CBIE/SBIE, Computer \& Education e Higher Education.

\subsection{Tipos de avaliações}

Na maioria dos estudos (27 artigos, 59\%) foram utilizados questionários com o objetivo de avaliar a aprendizagem através de ambientes virtuais. Entretanto, somente em nove artigos (33\%) é apresentado um modelo de questionário. Destes nove, seis se baseiam no Modelo Savi (2011) (22\%) bastante conhecido nas avaliações de aprendizado que avalia não só a aprendizagem como também a experiência do usuário e a motivação. 
V Congresso Brasileiro de Informática na Educação (CBIE 2016)

Anais do XXVII Simpósio Brasileiro de Informática na Educação (SBIE 2016)

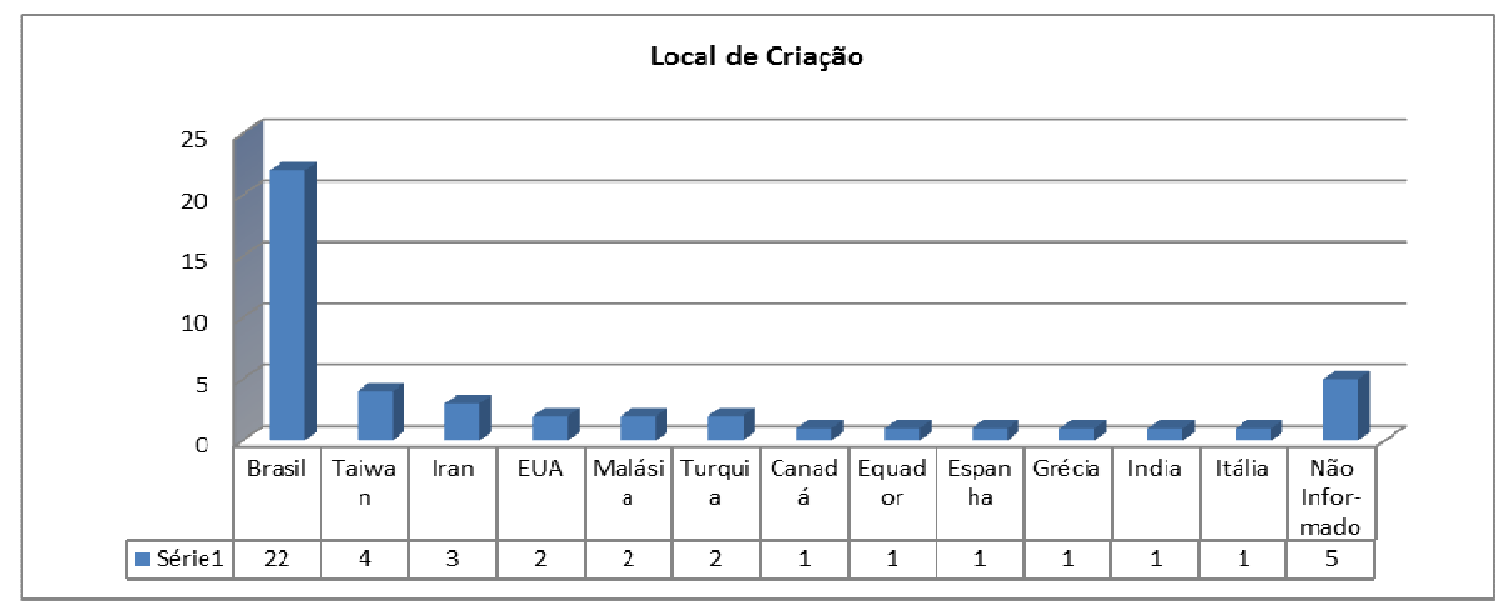

Figura 2. Local de Desenvolvimento dos Ambientes Virtuais

Dos estudos que utilizam questionário, 10 artigos (37\%) têm perguntas para avaliar a opinião do usuário não somente em relação à aprendizagem adquirida, mas também sobre outros aspectos como, por exemplo, o foco durante a atividade. As seguintes perguntas foram encontradas: (i) Que atividades você preferiu? [Ibanez, DiSerio e Delgado-Kloos 2014]; (ii) Que atividades não atraíram a sua atenção? [Ibanez, Di-Serio e Delgado-Kloos 2014]; (iii) Durante a partida, o jogo INDICASUS favoreceu ou dificultou a sua aprendizagem? [Costa, 2011]; (iv) As táticas do jogo favoreceram ou dificultaram sua aprendizagem durante a partida? Por quê? [Costa, 2011]; (v) O jogo ajudou a aprender novos conteúdos? [Savi, 2011] e (vi) O jogo ajudou a reforçar o conhecimento da disciplina? [Savi, 2011].

A maioria das perguntas $(63 \%)$ é feita com intuito de testar o ganho de conhecimento, servindo como uma ferramenta de avaliação. $71 \%$ destes trabalhos usaram técnicas de pré e pós-teste enquanto que os demais (29\%), apenas um pós- teste.

Apenas cinco estudos utilizaram a observação com o objetivo de avaliar o comportamento dos alunos em uso do ambiente virtual de aprendizagem. Apenas um trabalho [Levay et al, 2015] usou a observação com anotação e dois trabalhos [Costa, 2011], [Tsai et al, 2015] tiveram a necessidade de gravar a interação dos usuários. Dos trabalhos encontrados, 11 estudos relatam experimentos utilizando grupo de controle para verificar a eficácia da aprendizagem. Dos 11 artigos, em oito (73\%) foram utilizados métodos tradicionais de aprendizado com estes grupos para validar o uso dos ambientes virtuais como uma ferramenta significativa no processo de ensino aprendizagem. Apenas três trabalhos utilizaram o grupo de controle de forma diferente, entre elas, uma atividade prática parecida com o jogo sem elementos lúdicos [Silva et al, 2012], outro jogo sem relação com o jogo a ser avaliado [Gonçalves, Thiry e Zoucas, 2011] e, finalmente, a utilização de vídeos educacionais [Lin et al, 2013].

O trabalho (Kalelioglu e Yasemin, 2014] relata o uso da entrevista após a utilização de uma ferramenta. Professores escolheram quatro ou cinco estudantes de cada classe de acordo com o interesse e o desempenho apresentado no uso. A entrevista englobava, além da opinião do usuário sobre a ferramenta, outros aspectos mais específicos, como por exemplo, facilidade no uso, problemas encontrados na sua utilização, entre outros. 
V Congresso Brasileiro de Informática na Educação (CBIE 2016)

Anais do XXVII Simpósio Brasileiro de Informática na Educação (SBIE 2016)

\section{2. Área de desenvolvimento das avaliações encontradas}

Os 46 artigos que descrevem sobre a avaliação da aprendizagem em ambientes virtuais apresentam-se nas mais diversas disciplinas (Figura 3), dentre elas, destacam-se a computação com o maior número de trabalhos desenvolvidos $(21,8 \%)$, em seguida, a engenharia de software $(15,2 \%)$, ciências $(15,2 \%)$, matemática $(8,7 \%)$, enfermagem $(6,5 \%)$, física $(4,3 \%)$, entre outras áreas.

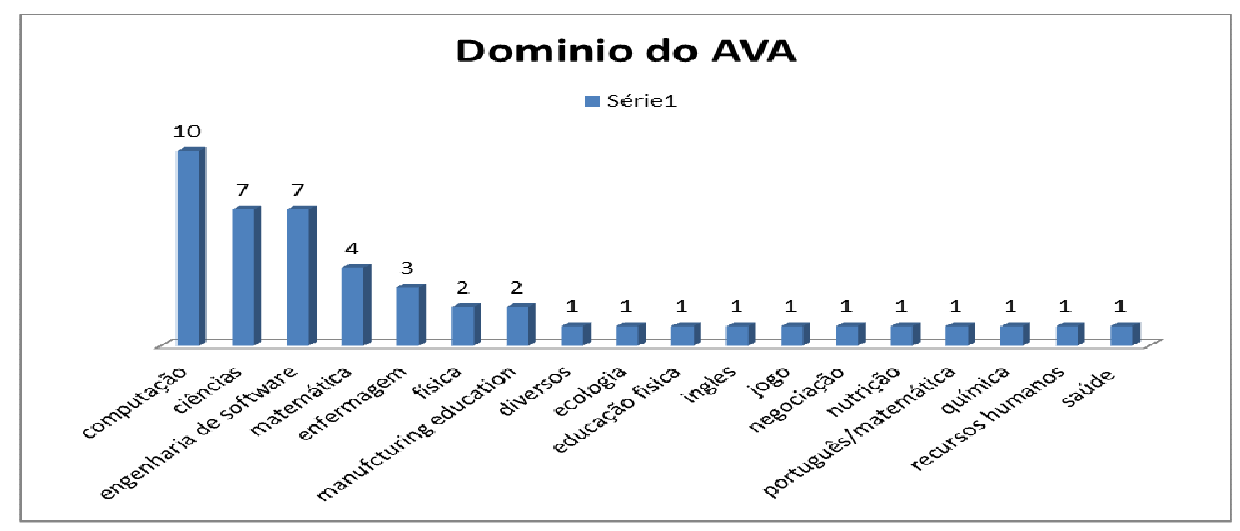

Figura 3. Área de Desenvolvimento dos Ambientes Virtuais

\subsection{Tipos de ferramentas utilizadas}

Dentre os estudos avaliados, 38 (83\%) são avaliações de aprendizagem utilizando um jogo como ferramenta de ensino. Este resultado deixa evidente a crescente utilização dos jogos para auxiliar no processo de aquisição do conhecimento. Em seguida, seis (13\%) artigos apresentam avaliação da aprendizagem em ambientes virtuais. Apenas dois trabalhos apresentaram imagens dos ambientes virtuais. Por último, dois (4\%) artigos relatam a avaliação da aprendizagem no uso de softwares. Os softwares encontrados não são mostrados, os autores apenas deixam por escrito seus objetivos.

\subsection{Fundamentação da proposta de avaliação}

Identificou-se, durante a revisão, que a maioria dos artigos (30 correspondendo a $65 \%$ ) utilizou uma proposta de avaliação criada pelo próprio autor. Nestes trabalhos não foram identificadas nenhuma citação referente ao modelo de proposta de avaliação. Dos 46 trabalhos sete (15\%) foram fundamentados no modelo de avaliação proposto por Savi (2011a) que é baseado nas estratégias de motivação de Arcs [Keller, 2010], no modelo de avaliação de Kirkpatrick (1994) e nos objetivos educacionais da Taxonomia de Bloom [BLOOM, 1956 in Savi, 2011a]. Destes sete artigos, 2 [Moreira e Coutinho, 2013] e [Silva et al, 2012] fizeram uma modificação no questionário proposto por Savi.

Um artigo [Neto, et al, 2015] usou, além do questionário padrão, um teste de matemática.Do restante dos artigos, seis (13\%) se baseiam em outros autores já citados no processo de avaliação da aprendizagem tais como Jorba e Sanmarti (2003), [Araujo et al, 2013], Ausubel (2003), [Gonzales, 2014], Liu e Lin (2009), [Liu et al, 2013], Liberali e Liberali (2011), [Levay et al, 2015], Malone e Leaper [1987, em Pourabdollahian, et al, 2012], Csikszentmihalyi [1992 em Pourabdollahian, et al, 2012] e Witmer e Singer (1998) [. Sabourin et al.2013]. E, finalizando, três (7\%) artigos [Gonzales et Rosa, 2014], [Neto et al, 2015], [Brito e Motta, 2015] fundamentam sua proposta unindo as próprias ideias com as de outro autor. 
V Congresso Brasileiro de Informática na Educação (CBIE 2016)

Anais do XXVII Simpósio Brasileiro de Informática na Educação (SBIE 2016)

\subsection{Público alvo dos Ambientes Virtuais}

Grande parte dos artigos (48\%) apresentam ambientes virtuais destinados a alunos do ensino superior de diversas áreas, sendo desenvolvido com alunos do curso superior em Ciência da Computação. Apenas um trabalho relatou a avaliação da aprendizagem no uso do ambiente virtual em uma empresa onde o público alvo eram engenheiros. $\mathrm{O}$ segundo maior público são os alunos do ensino fundamental (28\%), seguidos dos alunos do ensino médio (15\%).

\subsection{Tempo de duração das avaliações}

Dos trabalhos encontrados, 27 artigos (59\%) não descreviam a duração do processo de avaliação da aprendizagem proposto por eles. Dos artigos que apresentam esta informação, pode-se observar que o processo mais longo durou cerca de 12 meses para ser concluída e, o mais rápido, aproximadamente 2 horas. A durabilidade da avaliação é uma variável importante, pois em um trabalho [Neto, et al, 2015] é citado o fato de que os estudantes apresentaram fadiga na resolução do questionário e isso pode atrapalhar no processo de avaliação e acabar enviesando a pesquisa.

\subsection{Qualidade dos resultados das avaliações}

Os resultados identificados neste estudo apontam que as avaliações têm sido positivas em relação ao uso de ambientes virtuais como ferramenta no processo de ensino e aprendizagem. A maioria dos artigos $(40,87 \%)$ apresenta dados confirmando a eficácia do processo de aprendizagem. Apenas um artigo (2\%) não teve sua avaliação experimentada, os autores apenas apresentam um framework e uma proposta de efetivação. Dos trabalhos restantes, dois artigos (4\%) mostraram satisfação inconclusiva. Em um dos artigos [Carvalho Filho, 2011], o motivo seria o fato de que algumas atividades julgadas serem feitas sem compromisso por parte dos alunos, dificultando a análise dos resultados. No outro artigo [Aguiar, 2015] foi o fato de que o autor entende que cada aluno possui um estilo de aprendizagem e, parte deles pode ter se sentindo desconfortáveis com a metodologia utilizada para fazer a avaliação. Apenas em três artigos (7\%) não foi comprovada a satisfação com a avaliação feita, pois não foi observada diferença significativa da aprendizagem considerando a metodologia utilizada com os alunos que utilizaram o ambiente virtual.

\subsection{Visão Geral dos Resultados}

Os trabalhos encontrados na revisão sistemática realizam a avaliação da aprendizagem, ora através de questionários por meio de informações subjetivas, ora através de testes antes e depois da utilização do ambiente virtual. Foi identificado, na maior parte dos trabalhos, um impacto positivo no uso desses ambientes.

De um modo geral, existe uma preocupação em relação a maneiras para avaliar o impacto do uso dos ambientes virtuais na aprendizagem. Alguns dos artigos encontrados mostram que a utilização de um ambiente virtual como ferramenta de aprendizagem é bem aceita pelos estudantes, pois eles se sentem mais motivados a participar do processo de ensino. Em sua grande maioria, os trabalhos apresentavam questionários para obter informações sobre as características pessoais de cada aluno e a opinião em relação à atividade que haviam participado. Alguns trabalhos apresentaram variáveis que podem comprometer os resultados dos estudos que medem o aprendizado: a falta de uma pontuação significativa, estilo da aprendizagem, sexo dos jogadores, habilidade 
V Congresso Brasileiro de Informática na Educação (CBIE 2016)

Anais do XXVII Simpósio Brasileiro de Informática na Educação (SBIE 2016)

com jogos e habilidade com o computador. Estas variáveis precisam ter seu impacto sobre o processo de aprendizagem melhor investigadas. A amostra selecionada para realizar o experimento é considerada importante no processo de avaliação, pois alguns trabalhos indicavam ser importante aplicar novamente o experimento devido ao tamanho dela ou ao fato de não ser possível obter uma amostra aleatória.

\section{Conclusões}

Este trabalho apresentou uma revisão sistemática da literatura sobre a avaliação de AVAs. Com base no design adotado para a realização da revisão, verificou-se que diferentes visões, propostas ou modelos de avaliação de aprendizagem estão sendo propostos atualmente com o objetivo de observar o que tem sido desenvolvido para apoiar o processo de avaliação de ambientes educacionais. Em uma primeira análise, observa-se que a maioria dos trabalhos destacam ou o processo de desenvolvimento do ambiente virtual ou a avaliação do mesmo em relação a aspectos como interface, qualidade, clareza abstendo-se quase sempre de tratar sobre o real ganho no ensino.

Com base na revisão sistemática realizada, verifica-se que pelo menos três trabalhos se destacam: o modelo Arcs [Keller, 2010], o modelo de Kirkpatrick (1994) e o proposto por Savi $(2010 ; 2011 ; 2011 a)$. Keller (2010) concebeu o modelo Arcs (Atenção, Relevância, Confidência, Satisfação) que tem como objetivo empregar estratégias motivacionais no projeto de materiais educacionais. Este modelo pode ser utilizado para avaliar a motivação de estudantes quando utilizarem materiais educacionais. Kirkpatrick (1994) desenvolveu um modelo de treinamento composto por quatro níveis. Estes avaliam a reação, aprendizagem, comportamento e resultados. O modelo também pode ser usado para avaliar materiais instrucionais por meio dos níveis propostos. Savi et al (2011) propõe um modelo para avaliação de jogos educacionais baseado no modelo de avaliação de treinamentos de Kirkpatrick, nas estratégias motivacionais do modelo Arcs, na experiência do usuário e na taxonomia de Bloom.

O uso de AVAs como coadjuvantes do processo educacional já está consolidado e bem documentado. Desta forma, a motivação para a realização da RSL apresentada neste artigo, é, além de compor um estado da arte na área de avaliação de AVAs, incentivar outros pesquisadores em informática na educação a desenvolverem propostas e modelos de avaliação, bem como divulgarem os resultados de seus processos avaliativos.

\section{Referências}

Aguiar, Janderson (2015) "Experiência baseada em Gamificação no Ensino sobre Herança em Programação Orientada a Objetos", In: Anais Workshops do Congresso Brasileiro de Informática na Educação, Vol. 4,. No. 1.

Araujo, Glauber G., e Aranha, E.H. (2013), "Avaliação formativa da aprendizagem com instrumentação em Jogos digitais: Proposta de um framework conceitual", In: Anais dos Workshops do Congresso Brasileiro de Informática na Educação, Vol. 2, No. 1.

Ausubel, D. P. (2013), "Aquisição e retenção de conhecimentos: uma perspectiva cognitiva", Lisboa. Plátano.

Bezerra, C. I. M. ; Coutinho, E. F. (2013), "Avaliação do Jogo iTestLearning: Um Jogo para o Ensino de Planejamento de Testes de Software", In: Anais do XXI Workshop sobre Educação em Computação (WEI), Maceió. 
V Congresso Brasileiro de Informática na Educação (CBIE 2016)

Anais do XXVII Simpósio Brasileiro de Informática na Educação (SBIE 2016)

Brito, W.A.T. ; Motta, C.L.R. (2014), "Recomendação de Jogos na Aprendizagem da Matemática baseado na Análise Diagnóstica e Teoria de Resposta ao Item", In: Anais do 25o Simpósio Brasileiro de Informática na Educação. Porto Alegre, RS, Sociedade Brasileira de Computação, v. 01. p. 934-943.

Carvalho Filho, C. G.(2011), "Utilização de Ambientes Virtuais de Aprendizagem para Ensino Médio: um estudo aplicado ao ensino de Ecologia", Dissertação de Mestrado profissional em Ensino de Ciências e Matemática. Pontifícia Universidade Católica de Minas Gerais.

Costa, K. C. M. da (2011), "Análise do jogo IN.DICA.SUS-o perfil da gestão em saúde: em busca da formação de profissionais críticos e reflexivos", Dissertação de Mestrado. Universidade Federal de Minas Gerais. Escola de Enfermagem.

Galdino, Danielle Patricia Nascimento, et al. (2014), "Avaliação da Aprendizagem a partir dos Materiais Didáticos Disponíveis no Ambiente Virtual de Aprendizagem", In: Anais do| XI ESUD - Congresso Brasileiro de Ensino Superior a Distância, 1835-1849.

Galvão, Maria Cecília Alves, et al. (2013), "Análise da Dimensão Didático-Pedagógica em Ambientes Virtuais de Aprendizagem." Revista Meta: Avaliação 5.13, 12-28.

Gomes, T. C. S., Barreto, P. P., Lima, I. R. A., Falcão T.P. (2015), "Avaliação de um Jogo Educativo para o Desenvolvimento do Pensamento Computacional na Educação Infantil", In: Anais dos Workshops do IV Congresso Brasileiro de Informática na Educação.

Gonçalves, Rafael Q., Thiry, M. e Zoucas, A. (2011), "Avaliação da Aprendizagem em Experimentos com Jogo Educativo de Engenharia de Requisitos", In: Anais do X Simpósio Brasileiro de Qualidade de Software (SBQS), Curitiba.

Gonzales, Eliéverson Guerchi, e Rosa, Paulo R. da Silva (2014), "Aprendizagem significativa de conceitos de circuitos elétricos utilizando um ambiente virtual de ensino por alunos da educação de jovens e adulto", In: Investigações em Ensino de Ciências 19.2, 477-504.

Jorba, J.; Sanmartí, N. (2003), “A função pedagógica da avaliação", In: BALLESTER, Margarida (Org.). Avaliação como apoio à aprendizagem, Porto Alegre, Artmed, 23-45.

Keller, John M. (2010), "The Arcs model of motivational design." Motivational Design for Learning and Performance, Springer US, 43-74.

Kirkpatrick, D. L. (1996). "Techniques for evaluating training programs", In: Classic writings on instructional technology, 1(192), 119.

Kitchenham, B. (2007), "Guidelines for performing Systematic Literature Reviews in software engineering", In: EBSE Technical Report EBSE-2007-01, 2007.

Levay, P., et al (2015), "Uma Experiência de Uso de Jogos Digitais como Ferramentas de Apoio para Aprendizagem de Inglês por Crianças", In: Anais do Workshop de Informática na Escola.Vol. 21.No. 1..

Liberali, Fernanda Coelho e Liberali, André Ricardo Abbade (2011), "Para pensar a metodologia de pesquisa nas ciências humanas", In: Inter-Faculdades Integradas Coração de Jesus, Rede Salesianas de Ensino, Vol. 1, n. 1, 17-33,

Lin, Chun-Hung, et al. (2013), "Game-Based Remedial Instruction in Mastery Learning for Upper-Primary School Students", In: Educational Technology \& Society 16.2, 271-281.

Liu, E.Z.F. and Lin, C.H. (2009), "Developing evaluative indicators for educational computer games", In: British Journal of Educational Technology, 40 (1), 174-178

Liu, E., Feng,Z and Chen,K. (2013), "The Effect of Game-Based Learning on Students' Learning Performance in Science Learning-A Case of Conveyance Go", In: Procedia-Social and Behavioral Sciences, 103, 1044-1051. 
V Congresso Brasileiro de Informática na Educação (CBIE 2016)

Anais do XXVII Simpósio Brasileiro de Informática na Educação (SBIE 2016)

Mattos, T. A.F., e Costa, J. W. da (2013), "Avaliação da aprendizagem: do papel para o computador", In: Anais dos Workshops do Congresso Brasileiro de Informática na Educação. Vol. 2, No. 1.

Neto, S., et al. . (2015), "Avaliação do Jogo Educativo Mundo de Euclides: Uma abordagem Multi-Perspectiva", In: Anais dos Workshops do Congresso Brasileiro de Informática na Educação. Vol. 4, No. 1.

Peixoto, K.S.M., Silva, R.A.R. and Costa, R.H.S. (2015), "Nursing Diagnoses in People with Acquired Immune Deficiency Syndrome: An Integrative Review of the Literature", In: Revista de Pesquisa: Cuidado é Fundamental (Online), 7, 2123-2136.

Pourabdollahian, B., Taisch, M. and Kerga, E. (2012), "Serious games in manufacturing education: Evaluation of learners'engagement", In: Procedia Computer Science 15, 256-265.

Sabourin, Jennifer L., et al. (2013), "Understanding and predicting student self-regulated learning strategies in game-based learning environments", In: International Journal of Artificial Intelligence in Education 23, 1-4, 94-114.

Savi, R. (2011), "Avaliação de jogos voltados para a disseminação do conhecimento", Tese de Doutorado em Engenharia e Gestão do Conhecimento, Universidade Federal de Santa Catarina.

Savi, Rafael, Wangenheim, C. e Borgatto, A. (2011), "Um Modelo de Avaliação de Jogos Educacionais na Engenharia de Software", In: Anais do XXV Simpósio Brasileiro de Engenharia de Software (SBES 2011), São Paulo.

Savi, R.C.S., Wangenheim, C.G.V., Ulbricht,V., Vanzin, T. (2011), "Proposta de um Modelo de Avaliação de Jogos Educacionais", In: RENOTE. Revista Novas Tecnologias na Educação, v. 8, n. 3, 1-10.

Shukor, Nurbiha A., Zaidatun Tasir, e Henny Van der Meijden (2015), "An Examination of Online Learning Effectiveness Using Data Mining", In: Procedia-Social and Behavioral Sciences 172, 555-562.

Silva, J. C.; Sousa, S. P. A.; Kulesza, R.; Brito, A. V. (2012), "Uma avaliação do emprego do jogo Modelando como apoio ao ensino de Engenharia de Requisitos". In: Anais do XX Workshop sobre Educação em Computação (WEI'2012) Curitiba, Brasil.

Souza, T.I.A., Franco, A.O.R., Silva, T.E.V., Vasconcelos, F.H.L (2013), "Avaliando o Desempenho discente em um AVA: Um Estudo de Caso Utilizando Estatística Multivariada", In: Anais do II Congresso Brasileiro de Informática na Educação (CBIE).

Tsai, Meng-Han, et al. (2015), "The effectiveness of a flood protection computer game for disaster education", In: Visualization in Engineering 3.1, 1-13.

Venancio, V., Lopes, R. D. "Avaliação da Aprendizagem em Sistemas Interativos: uma revisão comparativa focada no SBIE, WIE e WAvalia". Anais do SBIE 2013.

Witmer, B. G., and Singer, M. J. (1998), "Measuring presence in virtual environments: A presence questionnaire", In: Presence: Teleoperators and virtual environments 7.3, 225-240.

Wohlin, C., Runeson, P., Höst, M., Ohlsson M. C., Regnell B. e Wesslén, A. (2012), "Experimentation in Software Engineering", First Edition, Springer, Berlin, Heidelberg. 